\title{
Bacteriemia por Ochrobactrum anthropi en paciente en hemodiálisis por catéter tunelizado permanente. Caso clínico
}

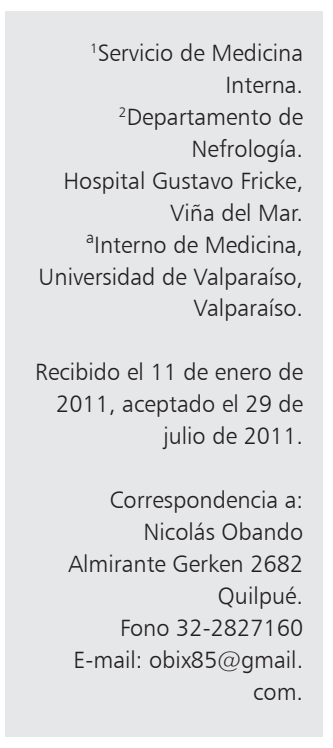

\author{
NICOLÁS OBANDO M. ${ }^{1, a}$, MARTA BADILLA S., ${ }^{1,2}$
}

\section{Catheter associated bacteremia caused by Ochrobactrum anthropi in a patient on hemodialysis. Report of one case}

Ochrobactrum anthropi is a rare pathogen in humans that is usually related to infections in inmunocompromised patients or people with indwelling medical devices. There are very few reported cases of $O$. anthropi bacteremia in patients on hemodialysis. We report a 19-year-old female on hemodialysis through a jugular catheter, with fever and malaise lasting one month. The catheter was withdrawn and Ochrobactrum anthropi was isolated from it and from blood cultures. The patient was successfully treated with levofloxacin.

(Rev Med Chile 2011; 139: 1344-1346).

Key words: Bacteremia; Catheters, indwelling; Hemodialysis; Ochrobactrum anthropi.
$\mathrm{O}$ chrobactrum anthropi es un bacilo Gram negativo, no fermentador, que se relaciona con infecciones en pacientes inmunocomprometidos o personas debilitadas con dispositivos sintéticos implantados. La revisión de la literatura en Medline describe solamente 4 reportes pre$\operatorname{vios}^{1,3}$ de infecciones por este agente en pacientes en hemodiálisis. En este artículo describimos un caso de bacteriemia en una paciente joven recibiendo hemodiálisis por catéter permanente tunelizado.

\section{Caso clínico}

Paciente de 19 años, de sexo femenino, con antecedentes de hipotiroidismo y síndrome nefrótico secundario a una glomeruloesclerosis focal y segmentaria, la que evolucionó a insuficiencia renal crónica terminal. Al no tener desarrollo de lecho vascular, se le instaló un catéter tunelizado permanente yugular, bajo condiciones asépticas en mayo de 2009, iniciando hemodiálisis trisemanal sin inconvenientes.
En junio de 2010 la paciente presentó un cuadro de un mes de evolución caracterizado por fiebre intra y post diálisis, con registros de hasta $39^{\circ}$ C, sin compromiso del estado general, a lo que se le agregó posteriormente, anemización, anorexia y franco compromiso del estado general. Al ingreso no se encontró foco infeccioso en los sistemas respiratorio, genitourinario y gastrointestinal y la piel en relación al catéter se encontraba sin secreción ni eritema. Dentro de los exámenes de laboratorio destacaba: anemia normocítica normocrómica con hemoglobina de $6,2 \mathrm{~g} / \mathrm{dl}$ y hematocrito de $17,9 \%$, leucopenia de $3.700 / \mathrm{mm}^{3}$ y proteína C reactiva de $2,8 \mathrm{mg} / \mathrm{dl}$. Ante la sospecha de infección de catéter vascular, se retiró el catéter tunelizado y se solicitó un cultivo con técnica Maki, además de dos hemocultivos periféricos, iniciándose tratamiento empírico con vancomicina.

Al tercer día, en ambos hemocultivos y en cultivo del catéter se aisló Ochrobactrum anthropi identificado en el equipo Vitek2 de Biomeriux (99\% de especificidad). En el antibiograma, el microorganismo resultó ser suceptible a amikacina, 
levofloxacino, gentamicina, meropenem e imipenem y resistente a ceftazidima, ampicilina/sulbactam, cefepime y aztreonam, por lo que se retiró el catéter y se inició tratamiento con levofloxacino. Evolucionó en buenas condiciones generales con disminución de los parámetros inflamatorios y sin secuelas a largo plazo. Actualmente, la paciente se encuentra en hemodiálisis por catéter en espera de acceso vascular definitivo.

\section{Discusión}

Ochrobactrum anthropi es un bacilo Gram negativo, aeróbico, no fermentador, móvil, oxidasa positivo. Pertenece a la familia Brucellaceae dentro de la clase Alphaproteobacteria ${ }^{4}$. El primer aislamiento de O. anthropi en muestras clínicas fue en $1988^{5}$. Su hábitat natural es el suelo y las fuentes de agua, frecuentemente se encuentra contaminando productos biológicos y esto puede ser causa de pequeños brotes ${ }^{6}$.

En los últimos años ha aumentado su importancia como patógeno oportunista en pacientes con compromiso inmunológico, especialmente en ambientes hospitalarios. La infección más frecuentemente relacionada a este microorganismo es la bacteriemia asociada a catéter intravascular, debido a la fácil adherencia del patógeno a materiales sintéticos, característica similar a los del género Staphylococcus ${ }^{7}$. Entre otras infecciones producidas por esta bacteria destacan: infecciones del tracto urinario, meningitis ${ }^{8}$, neumonía ${ }^{9}$, endocarditis ${ }^{10}$, endoftalmitis ${ }^{11}$ abscesos pelvianos, hepáticos y pancreáticos ${ }^{12} y$ artritis ${ }^{13}$. Desde entonces se ha considerado como agente de infecciones oportunistas en pacientes imunocomprometidos con catéter intravascular, sobre todo en personas con comorbilidad hematológica, neoplásica, trasplantados o en diálisis ${ }^{14}$.

Usualmente, las cepas de este agente son resistentes a los beta lactámicos como penicilinas, cefalosporinas y aztreonam; esta resistencia es mediada en parte por la presencia de beta lactamasas tipo Amp C inducibles. Son suceptibles a aminoglucósidos, fluoroquinolonas, carbapenémicos, tetraciclinas y trimetoprim-sulfametoxazol ${ }^{15}$.

Al hacer una revisión de la literatura, en Chile no se han reportado casos de infección por O. anthropi y en el mundo hay 4 reportes de infecciones de catéter vascular en pacientes en hemodiálisis por este patógeno, de los cuales uno es un paciente inmunocomprometido por el uso de prednisolona y ciclofosfamida ${ }^{1}$ y los otros 3 corresponden a pacientes diabéticos en hemodiálisis ${ }^{2,3}$. Aunque se considera a la insuficiencia renal crónica como un estado de inmunodeficiencia, se debe considerar la posibilidad de infección por $O$. anthropi en todos los pacientes que estén en hemodiálisis por catéter venoso incluso ante la ausencia de factores de riesgo.

En este caso la cepa aislada presentó resistencia a los betalactámicos, con un patrón de sensibilidad que coincide con lo descrito en las diferentes publicaciones ${ }^{10,16,17}$, por lo que es fundamental el antibiograma, dada la resistencia de este microorganismo frente a las cefalosporinas ampliamente usadas como tratamiento empírico.

Como conclusión debemos destacar el rol de Ochrobactrum anthropi como patógeno oportunista en pacientes en hemodiálisis, debiéndose tener en cuenta su aislamiento en cualquier muestra clínica y no sólo en infecciones hospitalarias o pacientes con factores de riesgo clásicos.

\section{Referencias}

1. Chertow G. Ochrobactrum anthropi bacteremia in a patient on hemodialysis. Am J Kidney Dis 2000; 35: E30.

2. Daxboeck F, Zitta S, Assadian O, Krause R, Wenisch C, Kovarik J. Ochrobactrum anthropi bloodstream infection complicating hemodialysis. Am J Kidney Dis 2002; 40: E17.

3. Javaid M, Rumjon A, Cubbon M. Ochrobactrum anthropi Bacteremia in a Non-Diabetic, Immunocompetent Hemodialysis Patient. Dialysis \& Transplantation 2008; 37: 452-3. doi: 10.1002/dat.20277.

4. Teyssier C, Marchandin H, Jean-Pierre H, Masnou A, Dusart G, Jumas-Bilak E. Ochrobactrum pseudintermedium sp. nov., a novel member of the family Brucellaceae, isolated from human clinical samples. Int J Syst Evol Microbiol 2007 57: 1007-13.

5. Holmes B, Popoff M, Kiredjian M, Kersters K. Ochrobactrum anthropi gen. nov., sp. nov. from Human Clinical Specimens and Previously Known as Group Vd Int J Syst Bacteriol 1988; 38: 406-6.

6. Scerckenberger P, Daneshvar M, Hollis D. Other nonfermentative Gram-negative rods. In: Murray P, editor. Manual of Clinical Microbiology 9th edition. ASM Press 2007; 770-94.

7. Saavedra J, Garrido C, Folgueira D, Torres MJ, Ramos JT. Ochrobactrum anthropi bacteremia associated with a 
catheter in an immunocompormised child and review of the pediatric literature. Pediatr Infect Dis J 1999; 18: 658-60.

8. Chang HJ, Christenson JC, Pavia AT, Bobrin BD, Bland LA, Carson LA, et al. Ochrobactrum anthropi meningitis in pediatric pericardial allograft transplant recipients. J Infect Dis 1996; 173: 656-60.

9. Gascón F, Zafra M, Castaño M, Cámara C. de la, Valle M, Sánchez I. Neumonía extrahospitalaria con bacteriemia por Ochrobactrum anthropi en un niño inmunocompetente. Rev Diagn Biol 2002; 51 (2): 69-70 .

10. Romero R, Peinado E, Sobrino D, Sáez N, Álvarez D, Peña G. Prosthetic mitral valve endocarditis due to Ochrobactrum anthropi: case report. J Clin Microbiol 2004; 42: 3371-3.

11. Braun M, Jonas JB, Schonherr U, Naumann GO. Ochrobactrum anthropi endophthalmitis after uncomplicated cataract surgery. Am J Ophthalmol 1996; 122: 272-3.

12. Vaidya SA, Citron DM, Fine MB, Murakami G, Goldstein EJ. Pelvic abscess due to Ochrobactrum intermedium [corrected] in an immunocompetent host: case report and review of literature. Clin Microbiol 2006; 44: 1184-
6. Erratum in J Clin Microbiol 2007; 45: 1672.

13. Battaglia TC. Ochrobactrum anthropi septic arthritis of the acromioclavicular joint in an immunocompetent 17 year old. Orthopedics 2008; 31 (6): 606.

14. Cieslak T, Robb M, Drabick C, Fischer G. Catheterassociated sepsis caused by Ochrobactrum anthropi: report of a case and review of related nonfermentative bacteria. Clin Infect Dis 1992; 14: 902-7.

15. Javaid M, Rumjan A, Cubbon M. Ochrobactrum anthropi bacteremia in a non-diabetic, immunocompetent hemodialysis patient. Dialysis \& Transplantation 2008; 37: 452-3.

16. Kettaneh A, Weill F, Poilane I, Fain O, Thomas M, Hocqueloux L. Septic Shock Caused by Ochrobactrum anthropi in an Otherwise Healthy Host. J Clin Microbiol 2003; 41 (3): 1339-41.

17. Bizet C, Bizet J. Comparative susceptibility of Ochrobactrum anthropi, Agrobacterium tumefaciens, Alcaligenes faecalis, Alcaligenes denitrificans subsp. denitrificans, Alcaligenes denitrificans subsp. xylosidans and Bordetella bronchiseptica against 35 antibiotics including 17 betalactams. Pathol Biol (Paris) 1995; 43 (4): 258-63. 